International Journal of Modern Physics A

(C) World Scientific Publishing Company

\title{
CP VIOLATION AND A SEARCH FOR NEW PHYSICS IN LHCb
}

\author{
LESLIE CAMILLERI* \\ PH Department, CERN, \\ CH-1211 Geneva 23, Switzerland \\ Leslie.Loris.Camilleri@cern.ch
}

\begin{abstract}
The physics program of LHCb concerns the study of CP violation in the B system, including more precise determination of the CKM matrix parameters and in particular the determination of $\phi_{s}$. The search for new physics which could manifest itself indirectly will be a high priority. The main advantages of $\mathrm{LHCb}$ over the $\mathrm{B}$ factories are the access to the $B_{s}$ system and very large B production rates. In addition, $\mathrm{LHCb}$ is a dedicated $\mathrm{B}$ physics experiment with excellent vertexing and particle identification capabilities. It is currently setting up its detector in its allocated LHC interaction region and is foreseen to be ready for data taking in November 2007 for the pilot run.
\end{abstract}

Keywords: LHCb; CP violation; B system, New Physics.

PACS numbers: 11.25.Hf, 123.1K

\section{Introduction}

Quark mass eigenstates differ from the weak (flavor) eigenstates and their mixing is described by a unitary matrix, V, the Cabbibo-Kobayashi-Maskawa (CKM) matrix ${ }^{1-2}$ such that:

$$
V V^{\dagger}=\left(\begin{array}{ccc}
V_{u b} & V_{u s} & V_{u b} \\
V_{c d} & V_{c s} & V_{c b} \\
V_{t d} & V_{t s} & t b
\end{array}\right)\left(\begin{array}{ccc}
V_{u d}^{*} & V_{c d}^{*} & V_{t d}^{*} \\
V_{u s}^{*} & V_{c s}^{*} & V_{t s}^{*} \\
V_{u b}^{*} & V_{c b}^{*} & V_{t b}^{*}
\end{array}\right)=\left(\begin{array}{lll}
1 & 0 & 0 \\
0 & 1 & 0 \\
0 & 0 & 1
\end{array}\right)
$$

This matrix is complex and includes a phase responsible for CP violation. In its Wolfenstein parametrization ${ }^{3}$ and to $O\left(\lambda^{5}\right)$, it can be expressed as:

$$
\left(\begin{array}{ccc}
1-\lambda^{2} / 2-\lambda^{4} / 8 & \lambda & A \lambda^{3}(\rho-i \eta) \\
-\lambda+A^{2} \lambda^{5}(1 / 2-\rho-i \eta) & 1-\lambda^{2} / 2-\lambda^{4}\left(1+4 A^{2}\right) / 8 & A \lambda^{2} \\
A \lambda^{3}(1-\rho-i \eta)+A \lambda^{5}(\rho+i \eta) / 2-A \lambda^{2}+A \lambda^{4}(1 / 2-\rho-i \eta) & 1-A^{2} \lambda^{4} / 2
\end{array}\right)
$$

The unitarity of the matrix implies six orthogonality relations between the matrix elements, which can be expressed as triangles in the complex plane. Two of the six triangles are particularly useful to describe B physics:

*On behalf of the LHCb collaboration. 


$$
V_{u d} V_{u b}^{*}+V_{c d} V_{c b}^{*}+V_{t d} V_{t b}^{*}=0 \quad V_{t b} V_{u b}^{*}+V_{t s} V_{u s}^{*}+V_{t d} V_{u d}^{*}=0
$$

They are displayed in Fig. 1 after dividing by $\left|V_{c b}^{*} V_{c d}\right|$. The angles $\beta, \gamma$ and $\chi$, shown in the figure, are related to the arguments of $V_{t d}, V_{u b}$ and $V_{t s}$ respectively.

The study of $\mathrm{CP}$ violation is motivated by the checking of the consistency of this interpretation and the measurement of the CKM parameters which are fundamental constants of nature. In addition New Physics (NP) could manifest itself indirectly in box or penguin diagrams in which the intermediate particles could be new ones in addition to the Standard Model (SM) ones. The possible results would be unexpected $\mathrm{CP}$ violation effects or unexpected properties of rare decays where SM contributions are small enough to allow NP effects to emerge.

\section{The production and detection of B particles}

In pp collisions B's are produced mostly through collision of partons carrying unequal fractions of the proton momenta. This results in both the $\mathrm{b}$ and $\bar{b}$ appearing near each other boosted either in the forward or backward direction. In order to capitalize on this, the LHCb detector is positioned in the forward direction and covers polar angles between 15 and $300 \mathrm{mrad}$. and a pseudo-rapidity range $1.9<\eta<$ 4.9. The $\mathrm{B}$ hadron production cross section above a transverse momentum, $\mathrm{p}_{T}$, of 2 $\mathrm{GeV} / \mathrm{c}$ is large, $230 \mu \mathrm{b}$. In order to maximize the probability of a single interaction per beam crossing, it was also decided to limit the luminosity in the LHCb interaction region to $\sim 2-5 \times 10^{32} \mathrm{~cm}^{-2} \mathrm{~s}^{-1}$ as opposed to the nominal LHC luminosity of $10^{34} \mathrm{~cm}^{-2} \mathrm{~s}^{-1}$, by not focussing the beams as hard. This has the additional advantage of limiting the radiation damage due to the high particle flux at small angles. Nonetheless as many as $10^{12} \mathrm{~b}$ pairs per year are expected to be produced in the $\mathrm{LHCb}$ region.

The characteristics of a B hadron are its long lifetime resulting in its decay far from the primary vertex and its high mass resulting in its decay products having a large $\mathrm{p}_{T}$. The signature for a $\mathrm{B}$ event is therefore particles with high $\mathrm{p}_{T}$ coming

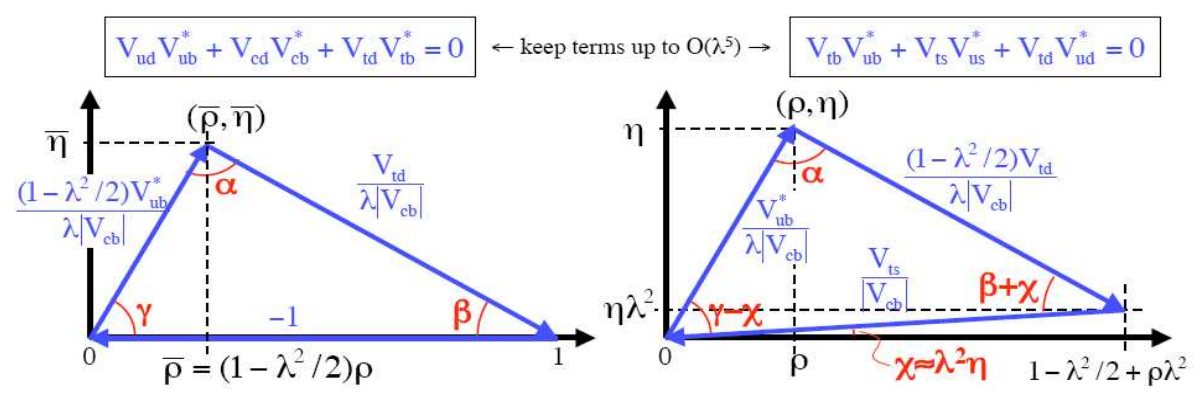

Fig. 1. The two unitarity triangles describing "Eq.(3)". 
from a displaced vertex. To know the flavor of the B at production the flavor of the companion (opposite side) $\mathrm{B}$ is determined by the charge of the kaon produced in the b to $\mathrm{c}$ to $\mathrm{s}$ decay chain, or of the lepton in semi-leptonic decays or of the whole accompanying $b$ jet. Alternatively the charge of the same side kaon often accompanying a $\mathrm{B}_{s}$, or of the pion resulting from a $\mathrm{B}^{* *}$ decay can also be used. The tagging power is $4-5 \%$ for a $\mathrm{B}_{d}$ and $7-9 \%$ for a $\mathrm{B}_{s}$.

\section{The $\mathrm{LHCb}$ detector and its performance}

The detector ${ }^{4-5}$ (see Fig. 2) is a $20 \mathrm{~m}$ long single arm spectrometer comprising a silicon vertex locator (VELO), followed by a first RICH counter, RICH1, a silicon trigger tracker, a 4Tm dipole, tracking chambers, a second RICH counter, RICH2, a preshower consisting of a 2.5 radiation length thick lead sheet sandwiched between two scintillator planes, a lead-scintillator shashlik geometry electromagnetic calorimeter, an iron-scintillator hadron calorimeter and a muon identifier.

The VELO consists of 21 stations located around the interaction region, each providing a measurement of the azimuthal and radial coordinates of tracks using, respectively, radial and annular strips. Each plane consists of two semi-circles which can be moved as close as $8 \mathrm{~mm}$ to the beams after the latter are declared stable. The measurement of the impact parameter of tracks relative to the primary vetex, a crucial parameter for identifying secondary vertices, is made with a precision of (14 $\left.+35 / \mathrm{p}_{T}\right) \mu \mathrm{m}$. RICH1 is meant to identify particles of low momenta and uses aerogel and $\mathrm{C}_{4} \mathrm{~F}_{10}$ radiators. RICH2, intended for higher momenta, uses $\mathrm{CF}_{4}$. In both counters, the Cerenkov light emitted by charged particles is focussed by a system of mirrors into rings onto planes of Hybrid Photo Diodes (HPD). Each HPD contains a 1024 pixel array that allows an accurate localization of the photons leading to a precise measurement of the radius of the rings. This measurement, combined with the measurement of momenta for which the precision is better than $0.5 \%$, allows

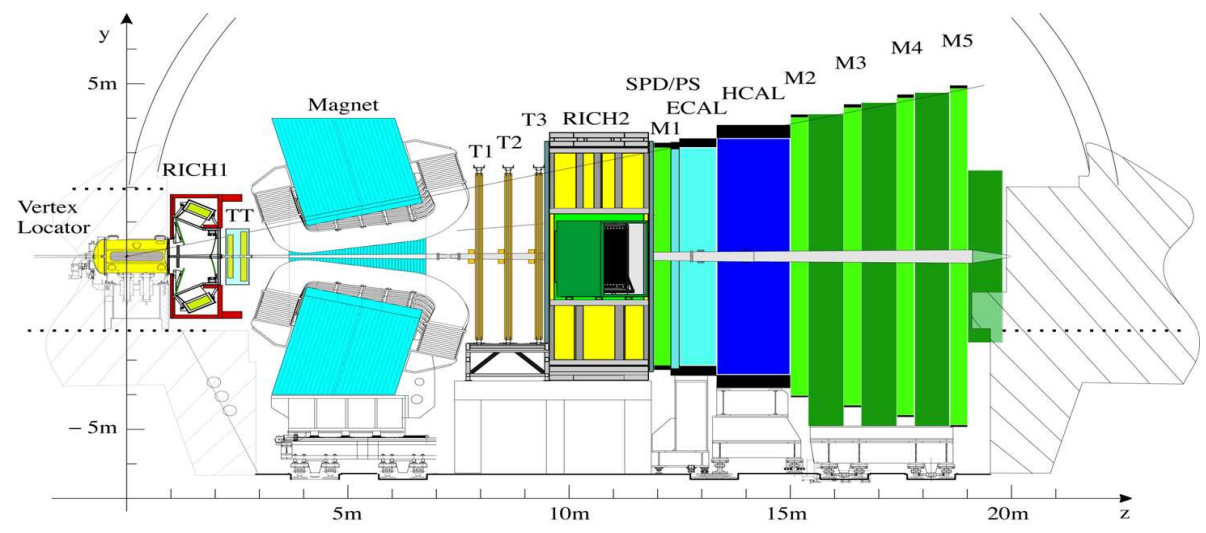

Fig. 2. The LHCb detector. 
particle identification with the efficiency and misidentification probability shown in Fig. 3. The majority of the detector is already installed and is foreseen to be complete by LHC turn on time.

\section{The trigger}

The LHCb trigger ${ }^{6}$ consists of two levels. The first level trigger, L0, is intended to reduce the $40 \mathrm{MHz}$ interaction rate to $1 \mathrm{MHz}$. This is achieved by using custom electronics that selects muons, electrons or hadrons above a given threshold in $\mathrm{p}_{T}$ varying between 1 and $4 \mathrm{GeV} / \mathrm{c}$. The $\mathrm{L} 0$ trigger uses information from the calorimeter and muon systems only and has a latency of $4 \mu \mathrm{s}$. The resulting events are then transmitted to a High Level Trigger (HLT) based on a processor farm. The HLT has the information from all the subdetectors at its disposal and, by successive application of algorithms, selects and records a total of $2 \mathrm{kHz}$ of the required event types.

\section{The measurement of $\phi_{s}$}

In the SM the $B_{s}-\bar{B}_{s}$ mixing phase, $\phi_{s}^{S M}=-2 \chi=-2 \lambda^{2} \eta$. This is a small quantity and indeed from UT fits a value for $\phi_{s}$ of $(-0.037 \pm 0.002)$ rad. is extracted ${ }^{7}$. However direct measurements are not very precise. Recently D0 has quoted ${ }^{8}$ a value of $\left(-0.79 \pm 0.56\right.$ (stat) ${ }_{-0.01}^{+0.14}$ (syst) $) \operatorname{rad} . \phi_{s}$ can be measured in the decay $B_{s} \rightarrow J / \psi\left(\mu^{+} \mu^{-}\right) \phi\left(K^{+} K^{-}\right)$which can proceed either directly or through $B_{s}$ $\bar{B}_{s}$ mixing. In the latter case the mixing box can include contributions from NP particles resulting in an additional contribution, $\phi_{s}^{N P}$, to $\phi_{s}$ such that it is no longer equal to $-2 \lambda^{2} \eta$. The procedure is to measure the proper time dependence of

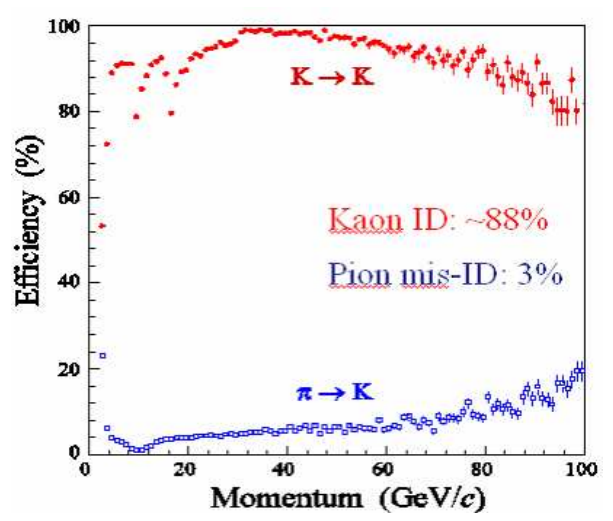

Fig. 3. The kaon identification efficiency (top curve) and pion misidentification probability(bottom curve) provided by the LHCb RICH counters. 
tagged $B_{s}$ and $\bar{B}_{s}$ and form the time dependent CP asymmetry which is given by:

$$
A_{C P}(t)=\frac{\eta_{f} \sin \phi_{s} \sin \left(\Delta m_{s} t\right)}{\cosh \left(\Delta \Gamma_{s} t / 2\right)-\eta_{f} \cos \phi_{s} \sinh \left(\Delta \Gamma_{s} t / 2\right)}
$$

where $\Delta m_{s}$ and $\Delta \Gamma_{s}$ are the difference in mass and width respectively of the two $B_{s}$ states and $\eta_{f}$ is +1 or -1 depending on the CP eigenstate. Because the final state contains two vector particles it is a mixture of both $\mathrm{CP}$ states. The contribution of these two states can be identified by doing an angular analysis based on the angle between the $\mu^{+}$and the normal to the $\phi$ decay plane. A simultaneous fit to both the angular and time distributions yields a precision ${ }^{9-10}$ on $\phi_{s}$ of 0.023 after a oneyear run amounting to $2 \mathrm{fb}^{-1}$. Analyzing pure CP states $\left(B_{s} \rightarrow J / \psi \eta, \eta_{c} \phi, D_{s} D_{s}\right)$ yields an additional measurement of $\phi_{s}$ with a precision of 0.059. Combining all the measurements results in a precision of 0.021 which is commensurate with the estimate of $\phi_{s}$ of -0.037 from UT fits. The NP contribution can be parametrized as

$$
M_{12}=\left(1+h_{s} e^{2 i \sigma_{s}}\right) M_{12}^{S M}
$$

where $M_{12}^{S M}$ is the dispersive part of the $B \bar{B}$ mixing amplitude in the SM.

Minimal Flavor Violation (MFV) implies no modification of the SM phase and $\sigma_{s}=0$, modulo $\pi / 2$. In Near Minimal Flavor Violation (NMFV) modification of the SM phase is allowed. Currently the $\operatorname{limit}^{11}$ on $h_{s}$ is still $<3.0$ and is not very constraining, but with $2 \mathrm{fb}^{-1}$ of LHCb data this limit could be improved to 0.1 .

\section{New Physics through Tree-Penguin Comparisons}

Whereas tree diagrams are insensitive to NP, penguin diagrams can be affected by it through new particles participating in the loop. Thus a measurement of the same quantity through both tree and penguin processes can indicate the presence of unexpected effects. Indeed the value of the angle $\beta$ as measured through a tree process via the decay $B_{d} \rightarrow K_{s} J / \psi$ differs from the value obtained through a penguin process such as the decay $B_{d} \rightarrow \phi K_{s}$. The difference ${ }^{12}, \delta \beta$, of $8^{\circ}$, amounting to $2.6 \sigma$, could be evidence for NP. The very same gluonic penguin loop occurs in $B_{s} \rightarrow \phi \phi$. Thus measurements of the angle $\chi$ as measured through this decay and through the tree process $B_{s} \rightarrow \phi J / \psi$ should again differ if NP is responsible for the angle $\beta$ difference. The penguin decay can proceed either directly or through mixing and since both the decay and the mixing terms depend on $V_{t s}$, the ratio of the $\bar{B}_{s}$ and $B_{s}$ decays to $\phi \phi$ is proportional to the product of mixing and decay terms $\left.\left.\left(V_{t b} V_{t s}^{*}\right) / V_{t b}^{*} V_{t s}\right)\left(V_{t b}^{*} V_{t s}\right) / V_{t b} V_{t s}^{*}\right)$ which is close to unity. CP violation effects are therefore expected to be less than $1 \%$ in the SM and this mode is therefore appropriate for searching for NP effects. Since the decay involves two vector particles, an angular analysis is required as well as a time-dependent one to extract the $\mathrm{CP}$ asymmetry. The proper time resolution for this decay is $42 \mathrm{fs}$ and for $2 \mathrm{fb}^{-1} 4000$ events are expected with a background/signal (B/S) estimate of $0.4<\mathrm{B} / \mathrm{S}<2.1$ at $90 \%$ C.L.. This would result in a sensitivity ${ }^{13}$ on a possible CP violating NP phase, $\phi^{N P}$, of $6^{\circ}$, to be compared to the $\delta \beta$ difference of $8^{\circ}$ observed in the $B_{d}$ system. 
$\mathrm{LHCb}$ is expected to run for longer than a single year and a data sample amounting to $10 \mathrm{fb}^{-1}$ can therefore be envisaged. This would improve the sensitivity on $\phi^{N P}$ to $2^{\circ}$.

\section{The Measurement of $\gamma$}

LHCb intends to measure $\gamma$ using several methods listed in Table 1. Combining all the measurements, the expected precision for a $2 \mathrm{fb}^{-1}$ data sample is $\sigma(\gamma) \sim 4^{o}$.

Table 1. The processes to be used by LHCb to measure the angle $\gamma$ and their expected precision.

\begin{tabular}{|c|c|c|c|}
\hline B mode & $\mathrm{D}$ mode & Method & $\sigma(\gamma) 2 \mathrm{fb}^{-1}$ \\
\hline$B^{+} \rightarrow D K^{+}$ & $K \pi+K K / \pi \pi+K 3 \pi$ & $\mathrm{ADS}^{14}+\mathrm{GLW}^{15-17}$ & $5^{o}-15^{o}$ see Ref. 18 \\
\hline$B^{+} \rightarrow D^{*} K^{+}$ & $K \pi$ & $\mathrm{ADS}^{14}+\mathrm{GLW}^{15-17}$ & Under study \\
\hline$B^{+} \rightarrow D K^{+}$ & $K_{s} \pi \pi$ & Dalitz $^{19-20}$ & $8^{o}$ see Ref. 21 \\
\hline$B^{+} \rightarrow D K^{+}$ & $K K \pi \pi$ & 4-body Dalitz ${ }^{22}$ & $15^{\circ}$ \\
\hline$B^{+} \rightarrow D K^{+}$ & $K \pi \pi \pi$ & 4-body Dalitz ${ }^{22}$ & Under study \\
\hline$B^{0} \rightarrow D K^{* 0}$ & $K \pi+K K+\pi \pi$ & $\mathrm{ADS}^{14}+\mathrm{GLW}^{15-17}$ & $7^{o}-10^{\circ}$ see Ref. 23 \\
\hline$B^{0} \rightarrow D K^{* 0}$ & $K_{s} \pi \pi$ & Dalitz $^{19-20}$ & Under study \\
\hline$B_{s} \rightarrow D_{s} K$ & $K K \pi$ & tagged, $\mathrm{A}(\mathrm{t}))^{24}$ & $13^{\circ}$ see Ref. 25 \\
\hline $\begin{array}{c}B_{d} \rightarrow \pi^{+} \pi^{-} \\
B_{s} \rightarrow K^{+} K^{-}\end{array}$ & & U-spin symmetry 26 & $4^{\circ}-10^{\circ}$ see Ref. 27 \\
\hline
\end{tabular}

Two of these methods will be described below.

\section{1. $\gamma$ from $B_{s} \rightarrow D_{s}^{+-} K^{-+}$}

This analysis is described in Ref. 25. A $B_{s}$ at production can decay to a $D_{s}^{-} K^{+}$ state either directly with an amplitude proportional to $V_{c b} V_{u s}$ or through mixing with an amplitude proportional to $V_{u b} V_{c s}$, as shown in Fig. 4.

Both these amplitudes are proportional to $A \lambda^{3}$ and, being of the same order of magnitude, lead to large interference effects. Since they are tree processes, they are insensitive to NP effects and yield a measurement of $\gamma$ which is independent of any such effects. Four decay time amplitudes need to be measured, $B_{s}$ and $\bar{B}_{s}$ decaying to either $D_{s}^{-} K^{+}$or to $D_{s}^{+} K^{-}$, yielding two asymmetries.

$$
D_{s}^{-} K^{+} \propto\left(\frac{V_{t b}^{*} V_{t s}}{V_{t b} V_{t s}^{*}}\right) e^{i \delta \Delta}\left(\frac{V_{u b} V_{c s}^{*}}{V_{c b}^{*} V_{u s}}\right) \propto e^{-i\left(\delta \Delta+\left(\gamma+\phi_{s}\right)\right)}
$$


and

$$
D_{s}^{+} K^{-} \propto\left(\frac{V_{t b} V_{t s}^{*}}{V_{t b}^{*} V_{t s}}\right) e^{i \delta \Delta}\left(\frac{V_{u b}^{*} V_{c s}}{V_{c b} V_{u s}^{*}}\right) \propto e^{-i\left(\delta \Delta-\left(\gamma+\phi_{s}\right)\right)}
$$

The precision with which $\mathrm{LHCb}$ can measure these asymmetries with a $10 \mathrm{fb}^{-1}$ data sample is shown in Fig. 5.

Fitting the time distributions together with the ones obtained through the ten times more abundant $B_{s} \rightarrow D_{s} \pi$ decay allows a simultaneous extraction of $\Delta m_{s}$, $\Delta \Gamma_{s}$ (the differences in mass and width of the physical states), the wrong tag rate (the probability that the tagging procedure yields an incorrect flavor assignment), the strong phase difference $\delta \Delta$ and $\left(\gamma+\phi_{s}\right)$. Using the value of $\phi_{s}$ obtained as described previously, $\gamma$ can be extracted with a precision of $13^{\circ}$ with $2 \mathrm{fb}^{-1}$ of data.
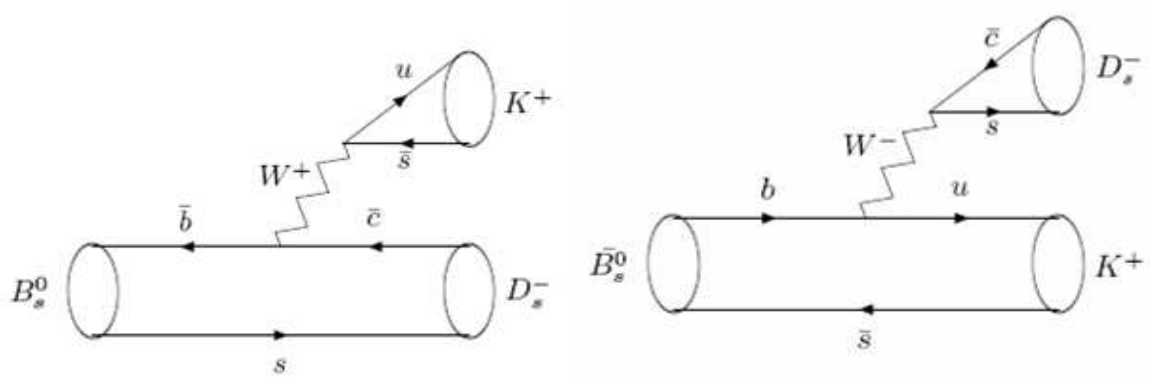

Fig. 4. The diagrams describing the $B_{s}^{0}$ and $\overline{B_{s}^{0}}$ decays to $D_{s}^{-} K^{+}$decays.

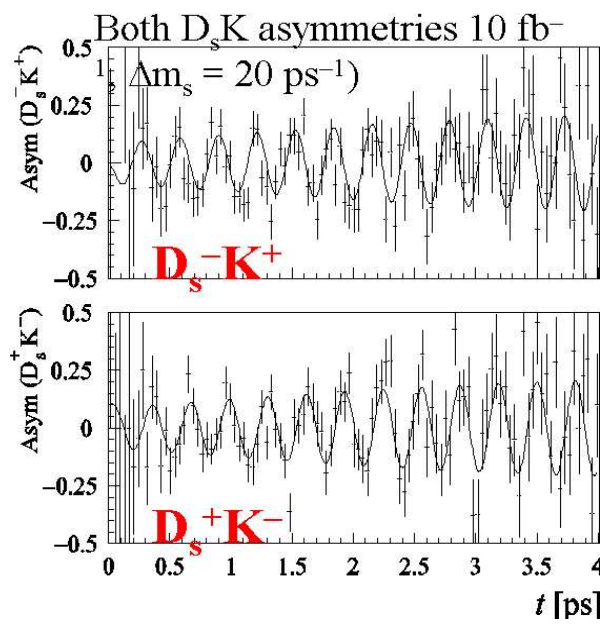

Fig. 5. The $D_{s}^{-} K^{+}$and $D_{s}^{+} K^{-}$asymmetries in $B_{s}^{0}$ and $\overline{B_{s}^{0}}$ decays as measured with a $10 \mathrm{fb}^{-1}$ data sample. 


\section{2. $\gamma$ from $B_{d} \rightarrow D K^{*}$}

This analysis is described in Ref. 23. The $B^{0} \rightarrow \bar{D}^{0} K^{* 0}$ and $B^{0} \rightarrow D^{0} K^{* 0}$ decays are both color suppressed as can be deduced from Fig. 6 .

They are therefore of comparable magnitude and again this leads to substantial interference effects. The method involves observing these two decays and the decay $B^{0} \rightarrow \bar{D}_{1} K^{* 0}$, where $D_{1}=\left(\bar{D}^{0}+D^{0}\right) / \sqrt{2}$, as well as the three charge conjugate reactions. The $D^{0}$ and $K^{*}$ are observed through their $K^{+-} \pi^{-+}$decay modes and the $D_{1}$ through its $\pi^{+} \pi^{-}$or $K^{+} K^{-}$decay. The flavor of the B is identified through the charge of the $\mathrm{K}$ in the $\mathrm{K}^{*}$ decay and that of the $\mathrm{D}$ by the charge of the $\mathrm{K}$ in its decay. They are therefore self tagging modes. Defining $\mathrm{A}_{1}, \mathrm{~A}_{2}, \mathrm{~A}_{3}$ as the magnitude of the three decay mode amplitudes and $\mathrm{A}_{4}$ as that for $\bar{B}^{0} \rightarrow \bar{D}_{1} \bar{K}^{* 0}$, then from the definition of $D_{1}$,

$$
A\left(B^{0} \rightarrow \bar{D}_{1} K^{* 0}\right)=\frac{1}{\sqrt{2}}\left[A\left(B^{0} \rightarrow \bar{D}^{0} K^{* 0}\right)+A\left(B^{0} \rightarrow D^{0} K^{* 0}\right)\right]
$$

leading to

$$
\frac{1}{\sqrt{2}} A_{3}=\left[A_{1}+A_{2} e^{i(\delta+\gamma)}\right]
$$

This expression, where $\delta$ is the strong phase, can be depicted as a triangle in the complex plane from which $\gamma$ can be extracted as shown in Fig. 7. Taking into account the doubly suppressed Cabbibo decays, the expected rates and B/S are listed in Table 2.

Taking the ratio of the favored to the suppressed amplitudes as 0.4 and letting the strong phase vary between $-180^{\circ}$ and $+180^{\circ}$, the sensitivity on $\gamma$ is $7^{0}-10^{0}$ for $2 \mathrm{fb}^{-1}$.

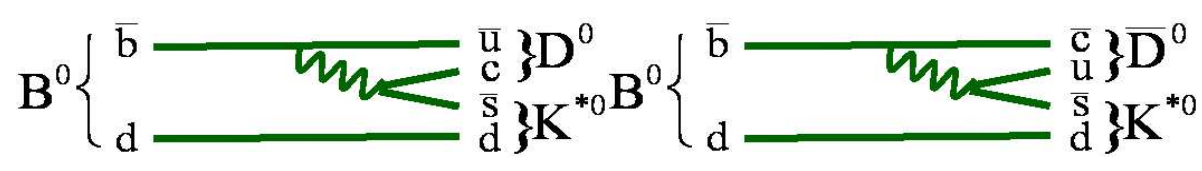

Fig. 6. The diagrams describing $B^{0} \rightarrow \bar{D}^{0} K^{* 0}$ and $B^{0} \rightarrow D^{0} K^{* 0}$ decays.

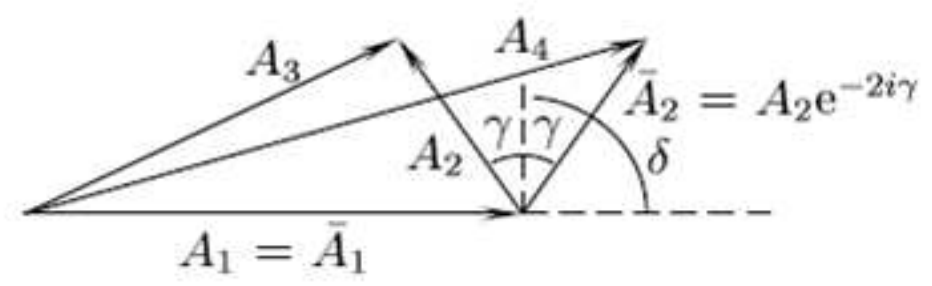

Fig. 7. The diagram relating the $\mathrm{A}_{1-4}$ amplitudes described in the text. 
Table 2. Event rates and background estimates for the decay $B_{d} \rightarrow D K^{*}$.

\begin{tabular}{cccc}
\hline B mode & Decay & Yield $/ 2 \mathrm{fb}^{-1}$ & $\mathrm{~B} / \mathrm{S}$ \\
\hline Favored & $B_{0} \rightarrow\left(K^{+} \pi^{-}\right)_{D} K^{*} 0+$ c.c. & 3400 & $<0.3$ \\
Suppressed & $B_{0} \rightarrow\left(K^{-} \pi^{+}\right)_{D} K^{*} 0+$ c.c. & 500 & $<1.7$ \\
& $B_{0} \rightarrow\left(K^{+} K^{-} / \pi^{+} \pi^{-}\right)_{D} K^{*} 0+$ c.c. & 600 & $<1.4$ \\
\hline
\end{tabular}

\subsection{The impact of the LHCb measurements of $\gamma$}

With a $10 \mathrm{fb}^{-1}$ data sample LHCb expects to measure the angles $\beta, \gamma$, and $\alpha$, with a precision of $\sigma(\sin (2 \beta))=0.01, \sigma(\gamma)=2.4^{0}$ and $\sigma(\alpha)=4.5^{0}$. LHCb will improve enormously on the knowlege of the angle $\gamma$ as measured with tree processes. This will allow to contrast this measurement with the estimates on $\gamma$ as measured with loop processes. The current precision on $\gamma$ with tree processes is shown in Fig. 8, together with the expected LHCb precision under two scenarii: agreement with loop processes or disagreement. The latter case would point to NP contributions.
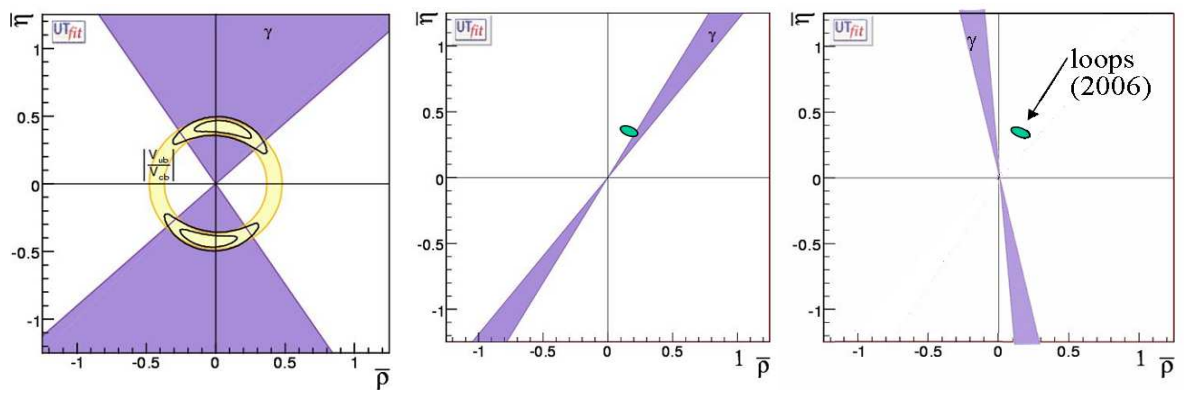

Fig. 8. A comparison between the angle $\gamma$ and the apex of the triangle as measured using loop processes in its current state (left plot) and after a $10 \mathrm{fb}^{-1}$ LHCb measurement of $\gamma$ using tree processes consistent (center plot) and inconsistent with the apex (right plot) as measured with loop processes.

\section{Rare decays}

\section{1. $B_{s} \rightarrow \mu^{+} \mu^{-}$}

This decay has a very small branching ratio ${ }^{28-29}$ in the SM: $(3.4 \pm 0.5) \times 10^{-9}$. The present limit from Tevatron measurements ${ }^{30-31}$ is $<7 \times 10^{-8}$ at $95 \%$ C.L. and will reach $<2 \times 10^{-8}$ with their expected full $8 \mathrm{fb}^{-1}$ data sample. The rate could be sensitive to NP through loops. As an example, the discrepancy in the measurement ${ }^{32}$ of the anomalous magnetic measurement observed at BNL can be explained ${ }^{33}$ in the Constrained Minimal Supersymmetric Standard Model (CMSSM) with a gaugino mass ranging from 250 to $650 \mathrm{GeV}$. With this same range of gaugino mass, the $B_{s} \rightarrow \mu^{+} \mu^{-}$branching ratio could be as high ${ }^{33}$ as $10^{-7}$, much higher than the SM 
predictions. Assuming the Standard Model branching ratio, a total of 70 events can be expected ${ }^{34}$ in a $2 \mathrm{fb}^{-1}$ exposure of $\mathrm{LHCb}$. The background is due mostly to a random combination of a $\mu^{+}$and a $\mu^{-}$originating from two distinct $\mathrm{B}$ decays. This is addressed by the very good mass resolution of $\mathrm{LHCb}, 18 \mathrm{MeV} / \mathrm{c}^{2}$ for this decay. Another background is from $\mathrm{B}$ decays into $\pi \pi, \pi \mathrm{K}$ or $\mathrm{KK}$ where the hadrons are misidentified as muons. This is addressed by the very good particle identification of $\mathrm{LHCb}$ and by its mass resolution. The LHCb sensitivity as a function of integrated luminosity is shown in Fig. 9. A three standard deviations observation at the level of the SM prediction could be made with $2 \mathrm{fb}^{-1}$ whereas a five standard deviation observation would require about $10 \mathrm{fb}^{-1}$.

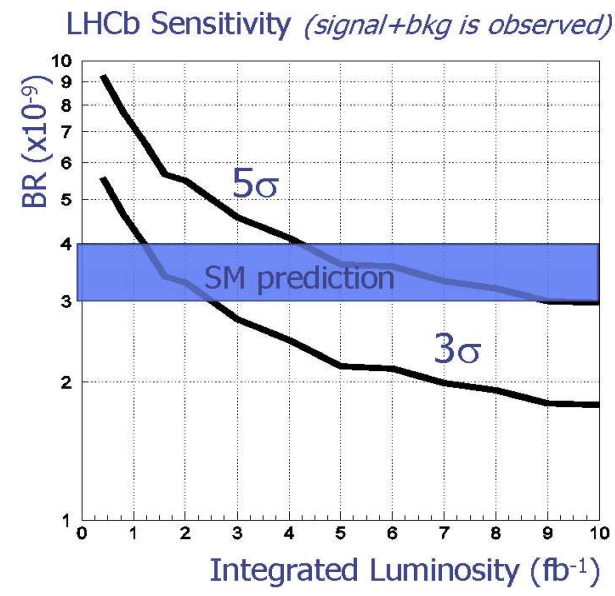

Fig. 9. The integrated luminosity required to achieve a three standard deviation (bottom curve) or five standard deviation (top curve) observation of the $B_{s} \rightarrow \mu^{+} \mu^{-}$decay as a function of its branching ratio. The standard model prediction, with its uncertainty, is also shown.

\subsection{Radiative decays}

In $\mathrm{b}$ to $\mathrm{q}$ transitions photons can be emitted from virtual $\mathrm{W}$ lines. They would be mostly left-handed in b decays and right-handed in $\bar{b}$ decays. Since radiative decays proceed through loop diagrams, they can again be sensitive to NP contributions ${ }^{35}$ which could modify the handedness of the interaction and hence the helicity of the photon. $B \rightarrow K^{*} \gamma, \phi \gamma, \Lambda_{b} \gamma$ are interesting decays to focus on. The photon helicity can be measured via conversions or in the parity-odd triple correlation between the momenta, $\mathrm{P}$, of the photon and of two out of the three hadrons in $B \rightarrow h_{1} h_{2} h_{3} \gamma$ : $P(\gamma) \cdot\left[P\left(h_{1}\right) \times P\left(h_{2}\right)\right]$. A possible such decay is $B \rightarrow K^{+} \pi^{+} \pi^{-} \gamma$. In addition, the observation of $\mathrm{CP}$ violation in $B_{s} \rightarrow \phi \gamma$, which should not occur if the helicities are as predicted in the SM, would also be an indication of NP. The sensitivity of LHCb to these radiative decays is currently being investigated ${ }^{36}$. 


\section{3. $B_{d} \rightarrow K^{*} \mu^{+} \mu^{-}$}

In this process, shown in Fig. 10, the radiated photon manifests itself as a $\mu^{+} \mu^{-}$ pair.

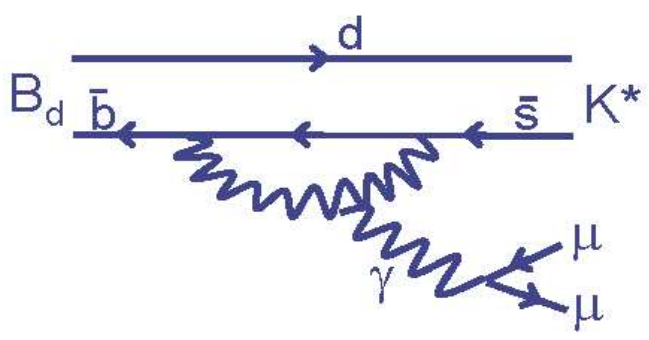

Fig. 10. The $B_{d} \rightarrow K^{*} \mu^{+} \mu^{-}$decay.

There will also be $Z^{0}$ contributions which will introduce some calculable righthanded contributions. These could be added to ${ }^{37}$ by New Physics in the form of gluino, chargino or neutralino loops followed by Higgs emission and decay to $\mu^{+} \mu^{-}$ or of a Higgs box. The result would be a modified angular distribution of the $\mu^{+} \mu^{-}$ pair. The branching ratio of the decay is $\left(1.22_{-0.32}^{+0.38}\right) 10^{-6}$, for which LHCb expects to measure $7200 \pm 2100$ events in a year, with the uncertainty mostly due to the branching ratio. The $\mathrm{B} / \mathrm{S}$ is calculated to be $<0.5$. The procedure ${ }^{38}$ is to measure the Forward-Backward Asymmetry (FBA) of the angular distribution of the $\mu^{+}$ relative to the $\mathrm{B}$ direction in the $\mu^{+} \mu^{-}$rest frame as a function of the $\mu^{+} \mu^{-}$invariant mass, as shown in Fig. 11 for a $2 \mathrm{fb}^{-1} \mathrm{LHCb}$ data sample.

The value, $\mathrm{s}_{0}$, of this invariant mass for which FBA is equal to zero is sensitive to NP contributions. $\mathrm{LHCb}$ is expected ${ }^{39}$ to achieve a precision of $0.52 \mathrm{GeV} / \mathrm{c}^{2}$ on $\mathrm{s}_{0}$ with $2 \mathrm{fb}^{-1}$, improving to 0.28 for $10 \mathrm{fb}^{-1}$.

\section{The next step}

$\mathrm{LHCb}$ is in the process of studying the possibility of running at ten times its design luminosity, namely $2 \times 10^{+33} \mathrm{~cm}^{-2} \mathrm{~s}^{-1}$, enabling it to accumulate a $100 \mathrm{fb}^{-1}$ data sample. The required upgrade of its detector would involve more selective trigger capabilities such as implementing a displaced vertex trigger at Level 0 , faster data acquisition and more radiation hard VELO sensors.

\section{Conclusions}

$\mathrm{LHCb}$ will be ready to collect data with its full detector as LHC turns on. The expectations are a pilot run at $900 \mathrm{GeV}$ center of mass energy late in 2007, followed by physics runs at $14 \mathrm{TeV}$ starting in 2008. It is set to make precision measurements 


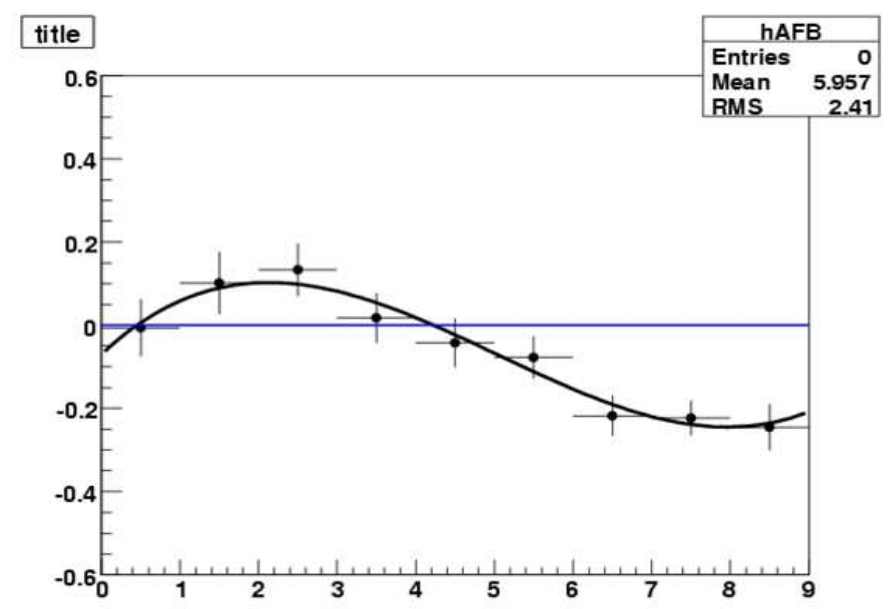

Fig. 11. An example of the precision obtainable with a $2 \mathrm{fb}^{-1} \mathrm{LHCb}$ data sample on the ForwardBackward asymmetry, defined in the text, in the $B_{d} \rightarrow K^{*} \mu^{+} \mu^{-}$decay, plotted as a function of the $\mu^{+} \mu^{-}$pair invariant mass.

that will severely constrain the unitarity triangle fits and probe rare decays. These measurements will either limit New Physics contributions to B decays or, more optimistically, uncover them.

\section{Acknowledgments}

I would like to thank all my LHCb collaborators, on behalf on whom I am giving this talk, for all their work and for their help in preparing this presentation. Many thanks also to the organizers of this symposium for a very well done job.

\section{References}

1. M.Kobayashi and T. Maskawa, Prog. Theor. Phys. 49, 652, 1973.

2. N. Cabibbo, Phys. Rev. Lett. 10, 531, 1963.

3. L. Wolfenstein, Phys. Rev. Lett. 51, 1945, 1983.

4. LHCb Letter of Intent, CERN-LHCC 1995-5.

5. LHCb Reoptimized TDR, CERN-LHCC 2003-030.

6. LHCb Trigger system, CERN-LHCC 2003-031.

7. M. Bona et al, hep-ph/0606167v2.

8. D0 Collab. (V.M.Abazov et al.), hep-ex/0701012v1.

9. L. Fernandez, EPFL Ph.D. Thesis 3613.

10. P. Vankov, LHCb Public Note 2007-65.

11. Z. Ligeti et al, Phys. Rev. Lett. 97, 101801 (2006) and hep-ph/0604112.

12. Heavy Flavor Averaging Group (E. Barberio et al.), http://www.slac.stanford.edu/xorg/hfag.

13. J. Libby, LHCb Public Note 2006-64.

14. D. Atwood et al, Phys. Rev. Lett. 78, 3257 (1997). 
15. M. Gronau and D. London, Phys. Lett. 253, 483 (1991).

16. M. Gronau and D. Wyler, Phys. Lett. 256, 172 (1991).

17. I. Dunietz, Phys. Lett. B270, 75 (1991).

18. M. Patel, LHCb Public Note 2006-66.

19. D. Atwood et al, Phys. Rev. D63, 036005 (2001) and hep-ph/0008090)

20. A. Giri et al, Phys. Rev. D68, 054018 (2003) and hep-ph/0303187)

21. V. Gibson et al, LHCb Public Note 2007-48 (in preparation).

22. J. Rademaker and G. Wilkinson, Phys. Lett. B647, 400 (2007).

23. M. Akiba and M. Gandelman, LHCb Public Note 2007-50 (in preparation).

24. R. Aleksan et al, Z. Phys. C54, 653 (1992) and hep-ph/0303187)

25. S. Cohen, LHCb Public Note in preparation.

26. R. Fleischer, Phys. Lett. B459, 306 (1999).

27. J. Nardulli, Talk given at CKM 2006 4th Workshop on the CKM Unitarity Triangle, Nagoya, Japan 12-15 December 2006,

28. A. J. Buras et al, Phys. Lett. B566, 115 (2003).

29. M. Misiak and J. Urban et al, Phys. Lett. B451, 161 (1999).

30. D0 Collab. (B. Aubert et al.), http://www.d0.fnal.gov/Run2Physics/WWW/results/b.htm.

31. CDF Collab. (A. Ambulencia et al.), Note 8176, http://www.cdf.fnal.gov/physics/new/bottom/0603016.blessed-bsmumu3.

32. Muon g-2 Collab. (G.W.Bennett et al.), Phys. Rev. Lett. 92, 161802 (2004).

33. J. Ellis et al JHEP 0502:013 (2005)

34. D. Martinez et al, LHCb Public Note 2007-033.

35. K. Kiers et al, Phys. Rev. D62, 116004 (2000).

36. D. Martinez et al, LHCb Public Note 2007-033.

37. D. Seidel el al., hep-ph/0106067.

38. B. Gristein and D. Pirjol, hep-ph/0505155.

39. J. Dickens, Talk given at CKM 2006 4th Workshop on the CKM Unitary Triangle, Nagoya, Japan, 12-16 December 2006 and J. Dickens et al, LHCb Public Note 2007-038.

40. R. Loren and D. B. Benson, J. Comput. System Sci. 27, 400 (1983).

41. OPAL Collab. (G. Abbiendi et al.), Eur. J. Phys. C 11, 217 (1999).

42. R. Loren and D. B. Benson, Introduction to String Field Theory, 2nd edn. (SpringerVerlag, New York, 1999).

43. R. Loren and D. B. Benson (eds.), Introduction to String Field Theory, 2nd edn. (Springer-Verlag, New York, 1999).

44. C. M. Wang, J. N. Reddy and K. H. Lee, New set of buckling parameters, in Shear Deformable Beams, ed. T. Rex (Elsevier, Oxford, 2000), p. 201.

45. R. Loren, J. Li and D. B. Benson, Deterministic flow-chart interpretations, in Introduction to String Field Theory, Ad. Series in Math. Phys., Vol. 3 (Springer-Verlag, New York, 1999), p. 401.

46. R. Loren, J. Li and D. B. Benson, Deterministic flow-chart interpretations, in Proc. 3rd Int. Conf. Entity-Relationship Approach, eds. C. G. Davis and R. T. Yeh (NorthHolland, Amsterdam, 1983), p. 421.

47. R. Loren, J. Li and D. B. Benson, Deterministic flow-chart interpretations, to appear in J. Comput. System Sci. 